\title{
Experimental Study on the Porosity Creep Properties of Broken Limestone
}

\author{
Shun-cai Li ${ }^{1,2, a}$, Qiang Li ${ }^{2}$, Jing-na Guo ${ }^{2}$ \\ ${ }^{1}$ School of Mechanical and Electrical Engineering, Jiangsu Normal University, Xuzhou, Jiangsu, 221116, China \\ ${ }^{2}$ State Key Laboratory for Geo-mechanics and Deep Underground Engineering, China University of Mining and Technology, Xuzhou \\ Jiangsu 221008, China
}

\begin{abstract}
In the underground engineering, the long-term stability of the surrounding rocks (especially the broken rocks containing water) and the ground settlement resulted from the seepage-creep coupling above goaf have been the important research subjects concerning the deep mining. For the broken rock, its porosity is an important structural parameter determining its creep properties, and the porosity change rate is more superior to describe the creep characteristics compared with the strain change rate at a certain direction. In this paper, MTS815.02 Rock Mechanics Test System is used to carry out the creep experiments on water-saturated broken limestone, and then the time curves of porosity and of the porosity change rate are obtained. By regression, we have got the relation equation between the porosity change rate with the instant porosity and the stress level during the creep. The study indicates that when the stress retains a constant level, the relation between the porosity change rate and the instant porosity can be fitted with a cubical polynomial. The obtained creep relation equation between the porosity change rate and the instant porosity and the instant stress provides a necessary state equation for studying the coupling between the seepage and the creep of the broken rock. Furthermore, the seepage in the broken rock has been verified to satisfy the Forchheimer's non-Darcy flow according to our previous studies, and its seepage properties, $k, \beta$ and $c_{a}$ can all be expressed respectively as the polynomial of the porosity, so, by combining with these three state equations we have obtained the four essential state equations for solving the coupling problems of the seepage and the creep for the broken rocks.
\end{abstract}

\section{Introduction}

As we know, the rock creep has great effect on the long time stability of the surrounding rock and surface subsidence. For broken rock, the porosity is the most basic factor determining its creep properties, but in the existing literature the stress and strain rate was mainly used to describe the creep properties of broken rock. For example, Wang ${ }^{[1]}$ carried out the numerical simulation on creep damage process of the rock surrounding the roadway under high stress in deep engineering of different depth; Zhu and $\mathrm{Ye}^{[2]}$ discussed the creep law affected by water content through the comparison of the rock creep test results under the dry and saturated state. Zhang and $\mathrm{Luo}^{[3]}$ studied on the rock creep characteristics under different stress level . Liu and Yang ${ }^{[4]}$ carried out tri-axial creep tests on the coal and rock through step loading method. $\mathrm{Li}^{[5]}$ and Zhang ${ }^{[6]}$ studied the creep test of marble and soft rock separately; A. k. Parkin ${ }^{[7]}$ used compression meter to study the rheological property of granular materials. Shen ${ }^{[8]}$ proposed three parameters model of rock-fill creep ${ }^{[9]}$ through the rheological experiments on limestone; Cheng ${ }^{[10]}$ proposed rock-fill creep model of nine parameters and obtained its parameter indexes through the tests. Liang ${ }^{[11]}$ pointed out that the creep law of rock-fill decays exponentially with time according to the tests; Guo ${ }^{[12]}$ proposed modified three-parameter rheological model of coarse granular

\footnotetext{
a Corresponding author: zsclsc@263.net
}

material; Wang ${ }^{[13]}$ summarized the rheological status of coarse-grained materials and pointed out that the experiment studies on granular materials were not sufficient. They proposed that it is necessary to study the micro deformation mechanism of coarse-grained materials in consideration of the scale effect of indoor test.

For the granular material, porosity is a physical quantity which can reflect the creep characteristics comprehensively. Usually, in the previous literature we can get the creep curve of the axial strain changing with time under each stress level, but the changing rules of porosity in the creep process have not been given directly.

This paper argues that the creep characteristics of rock are mainly determined by the current level of porosity and stress. The data of porosity changing can be achieved within a short time under the high porosity and high stress levels. As a result, the test time can be shortened and the cost can be reduced. In this paper, we carried out the creep tests on granular limestone saturated by water with MTS815.02 Rock Mechanics Test System ${ }^{[14][15]}$, then we got the relations among the change rate of porosity, the instant porosity and the stress level in the creep process.

\section{Test object and the scheme}

We carried out the experiments on the broken rock by the test system ${ }^{[14]}$, as shown in Figure 1. 


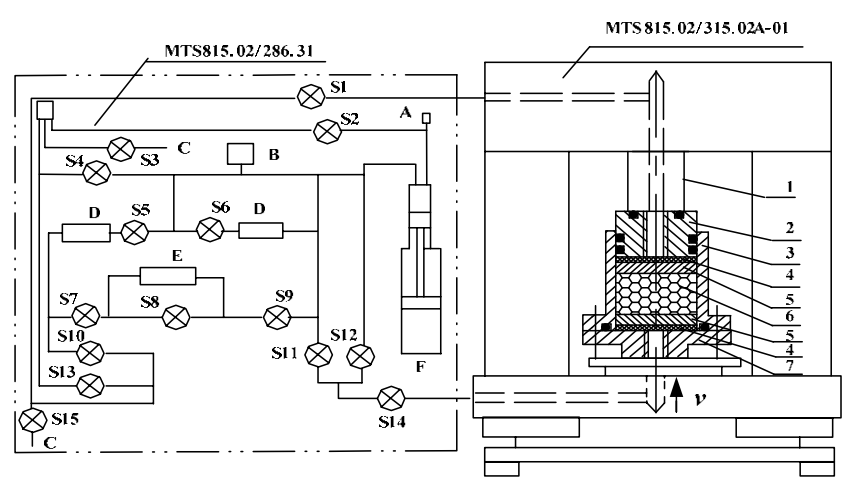

note: A-pressure transducer; B-pressure relief valve; $\mathrm{C}-$ drainage; D-stabilizer; E-differential pressure transducer; Fpressure intensifier (booster); S1-S15 are switches.

1-spherically seated upper plate for axial loading; 2-piston; 3cylinder tank; 4-felt; 5-permeable plate with many holes; 6rock sample; 7-base plate

Figure 1. MTS815.02 rock mechanics test system ${ }^{[14]}$

Test object is broken limestone with particle size 10 $\sim 15 \mathrm{~mm}$, and its mass density is $2700 \mathrm{~kg} / \mathrm{m}^{3}$, the axial compressive strength is $\sigma_{c}=62.3 \mathrm{MPa}$. Test scheme is as follows:

According to the increasing of the axial load, we set five stress levels. The corresponding axial load is $20 \mathrm{kN}$, $40 \mathrm{kN}, 80 \mathrm{kN}, 160 \mathrm{kN}$ and $320 \mathrm{kN}$ respectively, and the creep time of five stress level is 15 minutes, 30 minutes ,60 minutes, 120 minutes and 240 minutes respectively, as shown in Table 1

Table 1. each stress level and corresponding creep time

\begin{tabular}{|c|c|c|c|c|c|}
\hline level & 1 & 2 & 3 & 4 & 5 \\
\hline Force $/ \mathrm{kN}$ & 20 & 40 & 80 & 160 & 320 \\
\hline Loading time/s & 40 & 120 & 120 & 120 & 120 \\
\hline Creep time $/ \mathrm{min}$. & 15 & 30 & 60 & 120 & 240 \\
\hline Stress $\sigma / \mathrm{MPa}$ & 1.58 & 3.16 & 6.32 & 12.63 & 25.26 \\
\hline
\end{tabular}

The relationship between the porosity change rate and the instant stress and porosity can be regressed by two steps. Firstly, fit the relationship between the rate of change of porosity and the porosity ( rather than the initial porosity) under the fixed stress level, which gets $a_{0}, a_{1}, a_{2}$ and $a_{3}$. Secondly, fit the relationship between $a_{0}$, $a_{1}, a_{2}, a_{3}$ and the current stress (rather than the initial stress). As a result, we can save test time, and reduce the cost of test.

Test flowchart are as follows:

(1) Determine the initial porosity of the sample. Weigh the sample quality $m$, and by the density $\rho$ of core we can calculate the volume $V_{0}$ before the rock sample was broken for $V_{0}=\frac{m}{\rho}$. After the sample was placed into the cylinder, we can obtain the initial volume $V_{0}^{\prime}$ of the sample and the initial porosity $n_{0}$ by the height $h_{0}$ of sample in the cylinder and the inner diameter $2 a$ of the cylinder, we have

$$
V_{0}^{\prime}=\pi a^{2} \cdot h_{0}, \quad n_{0}=\frac{V_{0}^{\prime}-V_{0}}{V_{0}^{\prime}}
$$

(2) Saturate the rock sample;

(3) Apply the axial force gradually until its value reach the defined value, and keep the rock sample creep as the pre-designed time in Table 1. By record the height change $\Delta h$ of rock sample during the loading and creeping process, we can calculate the porosity

$$
n=\frac{\pi a^{2}\left(h_{0}-\Delta h\right)-\frac{m}{\rho}}{\pi a^{2}\left(h_{0}-\Delta h\right)}
$$

(4) Calculate the porosity change rate $\dot{n}$ at each sampling time by the finite difference method

$$
\left.\dot{n}\right|_{i+1}=\frac{n_{i+1}-n_{i}}{\Delta t}
$$

By regression, the relationship between $\dot{n}$ and $\dot{n}$ at each stress level is obtained by $n-\dot{n}$ scatter diagram, further more we can get therelationship between $a_{0}, a_{1}, a_{2}, a_{3}$ and the stress level.

$$
\dot{n}=a_{0}+a_{1} n+a_{2} n^{2}+a_{3} n^{3}
$$

\section{The test results}

\section{1 $n-t$ and $\dot{n}-t$ curves under various stress levels}

According to the collected axial displacement and the Equation (2), we can calculate the porosity of each time under each stress level. Thus we can draw time history curve of the porosity and porosity change rate under various stress levels, and draw relation curve of the porosity and the porosity change rate, as shown in Figure $1 \sim 10$ respectively. As a result, at the same stress level, the larger the porosity $n$, the greater the absolute of change rate $\dot{n}$. By regression analysis, we can obtain $\dot{n}-n$ relation curve of each stress level, which can be fitted by cubic polynomial.

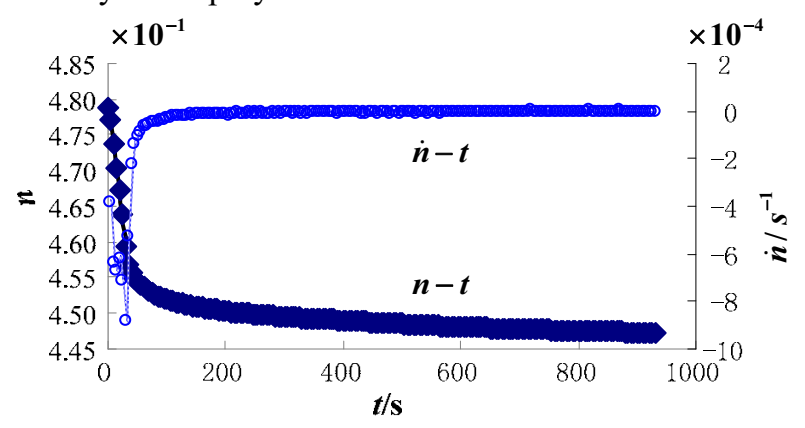

(a) $\dot{\boldsymbol{n}}-\boldsymbol{t}$ curve and $\boldsymbol{n}-\boldsymbol{t}$ curve 


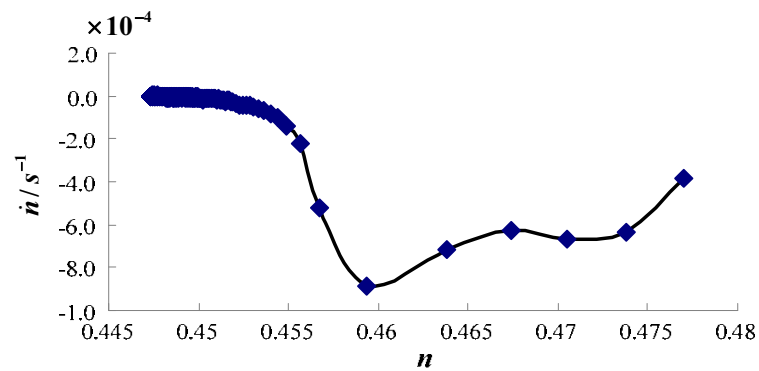

(b) $\dot{\boldsymbol{n}} \boldsymbol{-} \boldsymbol{n}$ curve

Figure 2. curves of porosity and its change rate at $\sigma_{a}=1.58$ $\mathrm{MPa}$

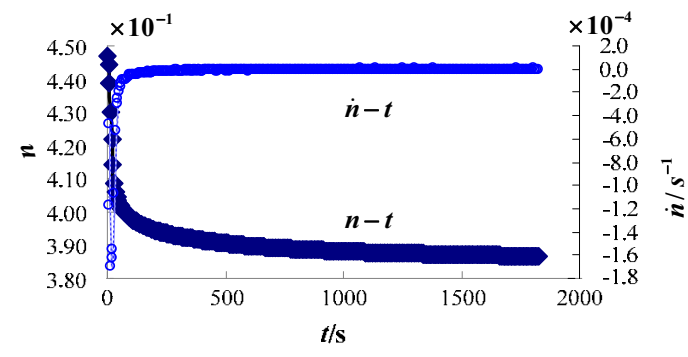

(a) $\dot{n}-t$ curve and $n-t$ curve

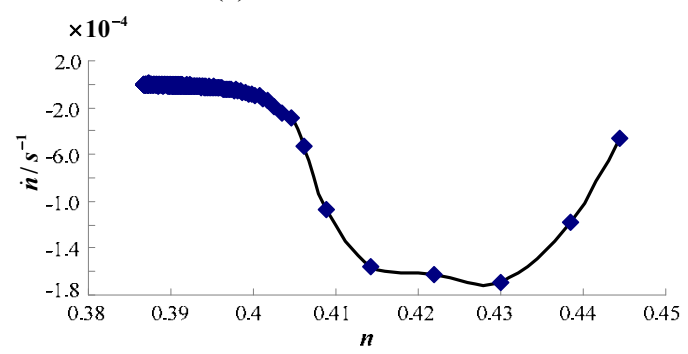

(b) $\dot{n}-n$ curve

Figure 3. curves of porosity and its change rate at $\sigma_{a}=3.16$ $\mathrm{MPa}$

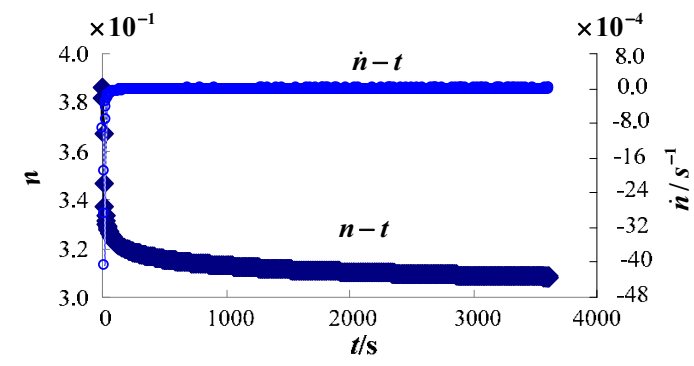

(a) $\dot{n}-t$ curve and $n-t$ curve

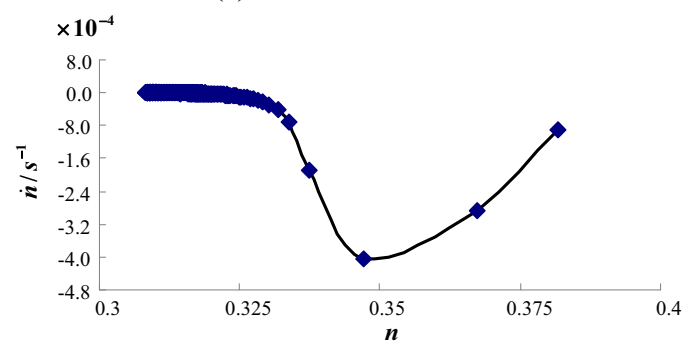

(b) $\dot{n}-n$ curve

Figure 4. curves of porosity and its change rate at $\sigma_{a}=6.32$ $\mathrm{MPa}$

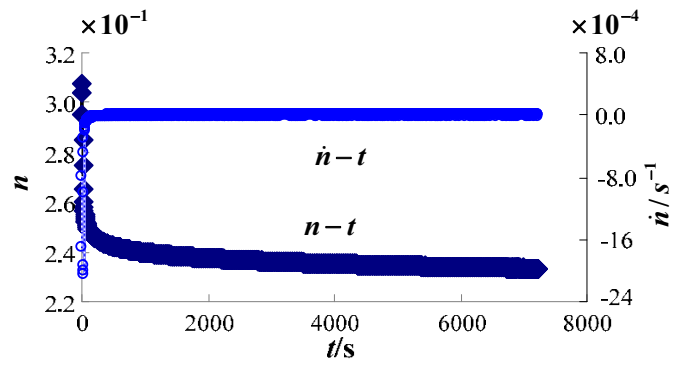

(a) $\dot{n}-t$ curve and $n-t$ curve

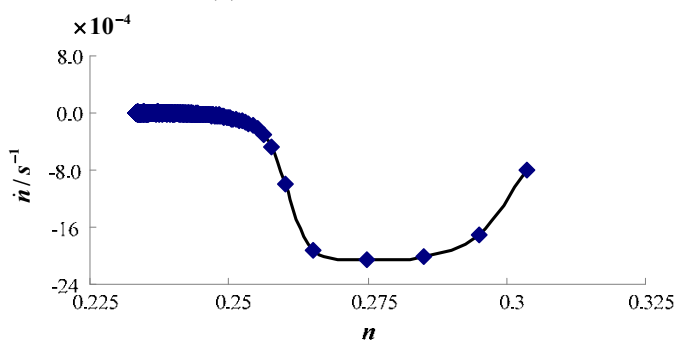

(b) $\dot{n}-n$ curve

Figure 5. curves of porosity and its change rate at $\sigma_{a}=$ $12.63 \mathrm{MPa}$

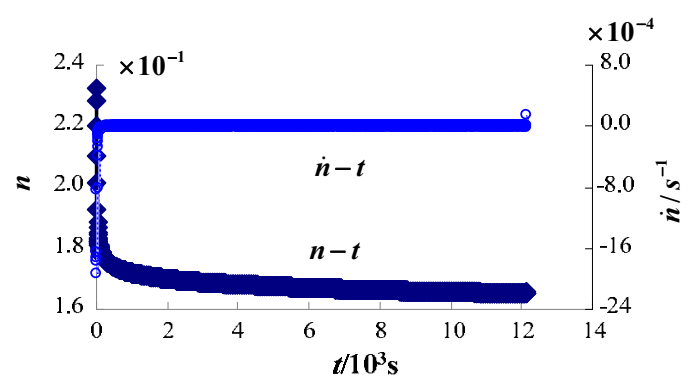

(a) $\dot{n}-t$ curve and $n-t$ curve

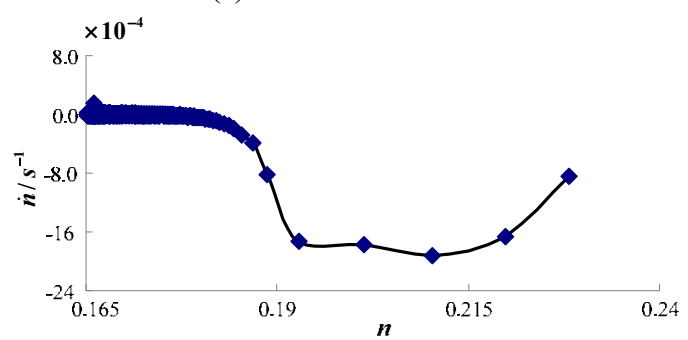

(b) $\dot{n}-n$ curve

Figure 6. curves of porosity and its change rate at $\sigma_{a}=25.26$ $\mathrm{MPa}$

Under various stress levels, the polynomial coefficients obtained by the curve fit are as shown in Table 2, where $\sigma_{a}$ is the axial stress and $\sigma_{c}$ is the uniaxial compressive strength of limestone core.

Table 2. coefficients of the cubic polynomials

\begin{tabular}{|c|c|c|c|c|c|}
\hline$\sigma_{a}(\mathrm{MPa})$ & $\sigma_{a} / \sigma_{c}$ & $a_{0}$ & $a_{1}$ & $a_{2}$ & $a_{3}$ \\
\hline 1.58 & 0.0254 & -18.6 & 122 & -265 & 192 \\
\hline 3.16 & 0.0507 & -4.41 & 32.4 & -9.3 & 64.5 \\
\hline 6.32 & 0.101 & -2.07 & 18.6 & -55.3 & 54.6 \\
\hline 12.6 & 0.202 & -0.566 & 6.57 & -25.2 & 32.0 \\
\hline 25.3 & 0.406 & -0.244 & 3.90 & -20.6 & 25.8 \\
\hline
\end{tabular}




\subsection{The relation equation between the porosity change rate and the present porosity and stress}

In Table 1 , polynomial coefficient $a_{0}, a_{1}, a_{2}, a_{3}$ is related to the ratio $\frac{\sigma_{a}}{\sigma_{c}}$ at various levels. By further regress we can get

$$
\begin{aligned}
& a_{0}=-0.0543\left(\frac{\sigma_{a}}{\sigma_{c}}\right)^{-1.5479} \\
& a_{1}=1.09\left(\frac{\sigma_{a}}{\sigma_{c}}\right)^{-1.2245} \\
& a_{2}=-7.22\left(\frac{\sigma_{a}}{\sigma_{c}}\right)^{-0.9029} \\
& a_{3}=11.8\left(\frac{\sigma_{a}}{\sigma_{c}}\right)^{-0.6806}
\end{aligned}
$$

Namely, the coefficients $a_{0} 、 a_{1} 、 a_{2} 、 a_{3}$ can be expressed by the power function of ratio $\frac{\sigma_{a}}{\sigma_{c}}$ respectively.

\section{The application of test results in the theoretical analysis and calculation}

The creep characteristics of rock (including broken rock) are determined by the rock pore and the fracture structure instead of the stress or strain of the rock in a certain direction. Therefore, the description of creep characteristics by porosity is superior to that by the stress or strain. When we study the seepage-creep coupling of the broken rock we can find that the mass conservation equation (continuity equation) and the momentum equation (equation of motion) can not constitute a set of complete control equation. In order to obtain a set of close control equation of seepage-creep system, we need to supplement the state equation. Equation (4) is actually a state equation on the porosity; it provides the basis for the analysis of coupled seepage-creep problems.

A large number of experiments prove that the rock seepage accord with the Forchheimer relationship ${ }^{[16]}$. There are three parameters to describe the seepage parameters: the permeability $k$, the non-Darcy flow factor $\beta$ and acceleration coefficient $c_{a}$. Although the logarithmic function, the power function and the exponential function can be used to fit the relationship between the seepage parameters and the porosity, there are some distinct errors. Only the polynomial fitting can get the best correlation. Certainly, the high order we fit the polynomial, the better effect we can get. However, this will complicate the analysis and the operation. Cubic polynomial can be chosen to give the following relationship.

$$
\left\{\begin{array}{l}
k=k_{0}\left(a_{0}+a_{1} n+a_{2} n^{2}+a_{3} n^{3}\right) \\
\beta=\beta_{0}\left(b_{0}+b_{1} n+b_{2} n^{2}+b_{3} n^{3}\right) \\
c_{a}=c_{a}^{0}\left(c_{0}+c_{1} n+c_{2} n^{2}+c_{3} n^{3}\right)
\end{array}\right.
$$

When we carry out numerical calculation, if the stress/strain state at the initial moments is known, we can use the strain state of a point to obtain porosity and determine the seepage parameters according to the Eq.(5), then calculate the porosity at the next moment according to the porosity creep equation (4), then calculate the stress/strain, pore pressure and seepage at the next moment according to the control equations. Cycle like this, we can get the dynamic stress/strain field for solid skeleton and dynamic seepage field

\section{Conclusions}

By the MTS815.02 rock mechanics testing system, we carried out the creep experiments on the water-saturated limestone at five levels of stress. As a result, we obtained the time curve and the relation curve of the porosity and its change rate. Then, we analyzed the test results and got the following conclusions:

(1) For broken rock the porosity change rate is more suitable to describe its creep properties than strain rate in a certain direction.

(2)The creep characteristic of broken rock is mainly determined by the porosity and the stress level, the greater the porosity and the higher the stress level, the greater the porosity change rate is.

(3)At the same stress level, we can use cubic polynomial to fit the porosity change rate and the current porosity; Under different stress levels, the polynomial coefficient depends on the ratio between the axial stress and the uniaxial compressive strength of the cores, and the coefficient can be fitted by the power function of this ratio.

(4) The obtained creep equation of the porosity change rate relating with the current porosity and stress provides an essential state equation for the study of creepseepage coupling effect.

\section{Acknowledgement}

The authors gratefully acknowledge the financial support from the National Natural Science Foundation of China (51574228, 51227003), Natural Science Foundation of Jiangsu Province University (14KJB440001).

\section{References}

1. Wang Y, Qi J, Yang C, Wei J. A study of nonlinear creep law in deep rocks. Rock and Soil Mechanics, 2005, 26(1): 117-121

2. Zhu H, Ye B. Experimental study on properties of rock creep in saturation. Chinese Journal of Rock Mechanics and Engineering, 2002, 21(12), 17911796 
3. Zhang Z, Luo J. Study on creep properties of rock under step load. Chinese Journal of Rock Mechanics and Engineering, 2004, 23(2), 218-222(in Chinese)

4. Liu J,Yang C, Li X. Jiang D. Testing study on creep of coal rocks in the tunnel of Wankai Speedway. Chinese Journal of Rock Mechanics and Engineering, 2004, 23(22), 3794-3798(in Chinese)

5. $\mathrm{Li} \mathrm{H}, \mathrm{Li} \mathrm{Z}, \mathrm{Su}$ C. Testing study on Creep characteristics of marble, Chinese Journal of Rock Mechanics and Engineering, 2004,23(22), 37453749(in Chinese)

6. Zhang X, Li Y, Zhang S, Huo B. Creep theory of soft rock and its engineering application, Chinese Journal of Rock Mechanics and Engineering, 2004,23(10), 1635-1639(in Chinese)

7. Parkin AK. Creep of rockfill (Part A). Maranhadas Neves E. Advances in rockfill structure. London: Kluwer Academic Publishers. 1992:221-239.

8. Shen Z, Zuo Y. Test research on the rheology character of rockfill [A]. The Sixth Proceedings of Geo-mechanics and Basic Engineering [C]. Shanghai: Press of Shanghai Tongji University, 1991, 443446(in Chinese)

9. Shen Z, Zhao K. Back analysis of creep deformation of rockfill dams. Journal of Hydraulic Engineering, 1998, (6):1-6

10. Cheng Z, Ding H. Creep test for rockfill. Chinese Journal of Geotechnical Engineering, 2004, 26(4): 473-476

11. Liang J, Liu H. Creep test for rockfill of CFRD. Chinese Journal of Geotechnical Engineering. 2002, 24(2):257-259(in Chinese)

12. Guo X, Wang D, Cai X, Dong L. Rheological analysis of concrete faced rock-fill dam. Journal of Hydraulic Engineering, 1999, (11): 42-46

13. Wang $M, H e X$, Cheng Z. Current situation and prospect of studies on rheology property of course stuff. Rock and Soil Mechanics, 2003(supp), 451-454

14. Liu Y, Li Y, Sun M. A new method of permeability test for loose rock. Ground pressure and strata control, 2002, 19(4):108-110

15. Liu W, Miao X, Chen Z. A Testing Method for Determining the Permeability of Over broken Rock. Journal of Experimental Mechanics, 2003, 18(1):5761

16. Chen Z. Bifurcation of Dynamic System of NonDarcy Flow in Post-Failure Rock [Ph. D. Thesis][D]. Xuzhou: China University of Mining and Technology, 2003 